\title{
HBIM AND THEMATIC MAPPING: PRELIMINARY RESULTS
}

\author{
D. Delpozzo ${ }^{1}$, D. Treccani ${ }^{1}$, L. Appolonia ${ }^{2}$, A. Adami ${ }^{1}$, B. Scala ${ }^{3}$ \\ ${ }^{1}$ He.Su.Tech. group, MantovaLab, Dept. of Architecture, Built environment and Construction engineering (ABC), Politecnico di \\ Milano, 46100 Mantua, Italy, damiano.delpozzo@mail.polimi.it, daniele.treccani@polimi.it, andrea.adami@polimi.it \\ ${ }^{2}$ RAVA, Piazza Severino Caveri, 11100 Aosta, Italy.1.appolonia@regione.vda.it \\ ${ }^{3}$ DICATAM, Università degli studi di Brescia, Via Branze 43, 25123 Brescia, Italy. barbara.scala@unibs.it
}

\section{Commission II}

KEY WORDS: HBIM, geometric modelling, Cultural Heritage, point cloud, mapping

\begin{abstract}
:
Geomatics' interest in Building Information Modelling (BIM) processes applied to Cultural Heritage is evolving in many directions. The traditional fields of Geomatics -data acquisition and processing- have been largely dealt with about HBIM (Historical Building Information Modelling) systems, but there are still some issues that need to be explored in more detail. In addition to modelling, it is essential to consider the information content of the model, how it is generated, recorded and managed.

If the objective of the HBIM model is the conservation project, it becomes essential to understand what types of data can be inserted in the model, how to record them and, above all, what their purpose is. The HBIM approach did not start out as a simple data repository, but as an information tool with the aim of helping the designer at all stages of the construction process. Moving to the field of preservation, much information about the building is represented by thematic maps. They allow to have a graphical image of the state of conservation of a façade or to understand the structural situation of a building.

This research, tested on the Arch of Augustus in Aosta, starts from the big amount of data acquired by the RAVA Laboratory of the Superintendency of Aosta during a long period. These data allowed to test different approaches to thematic mapping, according to the specific themes to be represented (previous restoration interventions, diagnostics, decay mapping, etc). Anyway, this experiment also required a theoretical reasoning that preceded the operational phases. Faced with a new system, in fact, it is always advisable to reason about the method applied, to avoid the error of simply translating a method that could instead be developed in new directions. The question, to which this article wants only to begin answering, concerns the role of thematic mappings in the preservation design made by a HBIM approach, their necessity and their implementation towards a truly three-dimensional data, which thus maintains all the information that is acquired directly in three dimensions and which instead, today, are reprojected in 2D or used as a placeholder in the $3 \mathrm{D}$ space
\end{abstract}

\section{INTRODUCTION}

Historic Building Information Modelling (HBIM) models, which by their very nature deal with the existing heritage of different cultural and historical value, have a strong documentary component. Since those models are not the product of design thinking (as for the case of new buildings), but they are used to describe something that pre-exists, the topic of managing building documentation is of prior importance. The documentation is here intended as the effective collection of information related to the building and its geo-referencing in the proper spatial position. In light of this, it is essential to find ways of reporting thematic information through $3 \mathrm{D}$ graphic models concerning materials, decays, results of specific analysis, diagnostics and also information about the development of the building or its previous restoration interventions.

However, this step gives rise to misunderstandings that do not always make the model effective or, at least, limit its potential use. Such misunderstandings derive from conceiving the thematic mappings just as a single graphical expedient and not as a design tool. Very frequently thematic mappings (e.g. the ones related to the actual state of conservation of materials of the elements of a building) may be very complex and not strongly connected to the object they are characterizing. For example, a mapping of decay of a masonry wall that is not directly linked to the wall itself is useful only as a representations tool; the relationships with the inner part of the structure or with the other facades remain some lacking information for the restorer.

In addition, from practice and from interviews with companies operating in the Architecture Engineering and Construction (AEC) sector, emerges that thematic mapping constitutes a double job, carried out firstly in the 2D environment according to traditional methods (i.e. using orthophotos in CAD environment), and secondly, reconstructing them in the 3D BIM Authoring environment exploiting various tailor-made methods. All these problems, and others later described, have been faced in the study of the Arch of Augustus in Aosta (Italy) in the research aimed to develop a new preservation project (Adami et al., 2021).

At the end of the modelling phase, through the construction of a federated model composed by survey model (a model made by point cloud or a mesh with a high level of accuracy) and conceptual model (where the elements are schemes, simplified versions of reality) model, it has been necessary to connect all the existing information to the conceptual model (Del Pozzo et al., 2022).

After the related works section, the paper described the methodology developed for the Arch of Augustus and, specifically, shows how each thematic map has been conceived in BIM approach, and how they can be used by the user as a tool for the next preservation project. A detailed part of the paper describes the script, written in Dynamo, which allows to retrieve previous graphical mapping of decay in the actual BIM environment, allowing not only to have significant 3D mapping 
but also the possibility of comparison between different state of decay.

\subsection{Evolution of thematic mapping}

This paper describes how the documental information related to thematic mapping of the Arch of Augustus were retrieved and stored directly in the 3D model on the 3D objects.

However, before delving into the technical details of the proposed method, it is necessary to reflect on the role of thematic mapping in a conservation project developed using an HBIM methodology.

The conservation project has its focal point in decay mapping. In fact, all conscious and practical decisions are made based on typologies of decay and his degree of urgency. For the designer it is also important to know decay's extension on the surface.

Traditionally, decay graphic representation in a twodimensional drawing is the most appropriate method to prepare a restoration project and, typically, it is made in CAD environment. In such a way, the extension of decay area can be easily computed, even if with discretion.

To cope with that, the designer increases the numbers of the areas, obtained from 2D drawings, of an arbitrary value expressed in percentage. The percentage is evaluated according to the complexity of the surface design the decoration: generally, between 10 to $30 \%$. This value, together with many other aspects that come from practice, will be discussed in a further multidisciplinary round table.

In a restoration project, the decay mapping cannot be avoided but it's needed to resolve the limits outlined above.

. The current way of mapping the degradation is the result of a long and slow evolution of the discipline of restoration and her relation to the world of CAD.

A principle to remember, before continuing the study of the ways to represent the mapping of degradation, is what now is meant decay?

Degradations are all the factors that can determine the equilibrium of matter. Consequently, they cause deteriorations of the conservative state of the heritage.

Initially, discipline of restoration represented pathologies in a realistic way on the two-dimensional design. Vegetation, loss, dust, stains, cracks and so on, made the expression of a decadent vision of monuments (Figure 1).

This procedure did not provide for the identification of defined areas. In fact, the aim was to represent the deterioration as in a photograph.

The use of hatching to indicate the deterioration has become a useful way to represent with greater scientific rigour the damages that have been called with names shared internationally. In addition, from this method of drawing the degradation with hatching is derived the way of drawing the conservation project.

Therefore, we are talking about an epochal step in the history of the conservation project.

HBIM, although introducing a three-dimensional representation, has not so far contributed to a more rigorous assessment of the extent of degradation. Nonetheless, we must not think that the BIM instrument is inadequate.

We know the difficulties modelling tools present to represent decays (Autodesk REVIT, Graphisoft ARCHICAD, and other BIM software) but if we want to use these, we will need to think in a new way about the objectives to be achieved in restoration project not only at the graphic level but also at the level of asset management.

In BIM model, approaches to drawing degradation are currently two types. On the one hand, BIM can indicate degradations as data's enrichment of constructive elements where degradations are located. A second way, it is to model decay extension, on the constructive element on which it is located. Both approaches have positive and negative aspects.

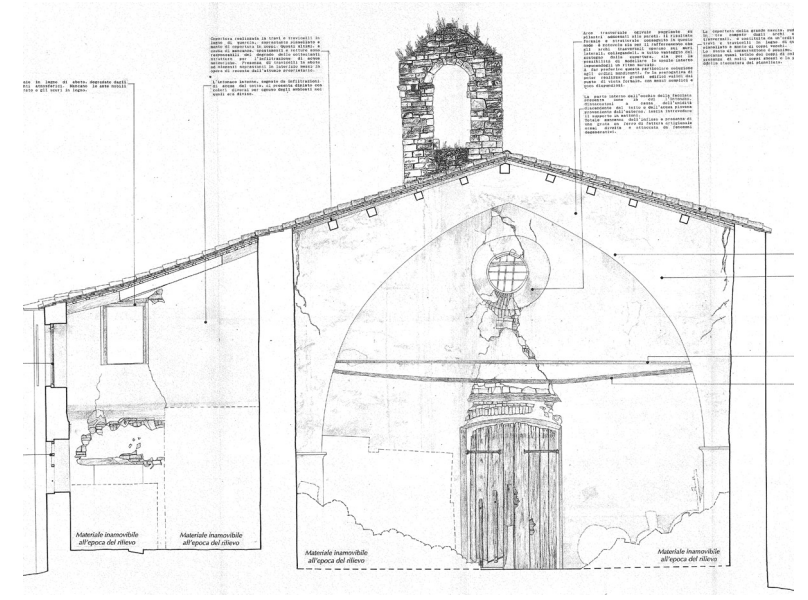

Figure 1: in old-style decay mapping, restorers did not use graphical areas and hatch to represent the state of conservation, but they preferred to draw the current situation with also the smallest details.

In the first approach, for example, thinking to some common approaches in Autodesk Revit, region command allows to identify an area, apply the degradation attribute. In opposite, the command doesn't allow visibility in $3 \mathrm{~d}$, so vanishes the additional interest in the use of this software and knowing its area.

The divide surfaces and paint command can be an adaptable method. The downside is the impossibility of mapping inclined surfaces. Even in this case, the software doesn't meet the expected integrative needs.

The creation of local models could be an additional solution. However, this way requires a lot of time to model, and it isn't an approach extensively usable in other monuments.

Another method is to create an adaptive generic model applicable to any surface with decay. In this case, starting from the orthophoto or from the cloud of points, decays are identified by a perimeter. This model is enriched with information, type of degradation, its degree of urgency and its extension.

This method is useful when the degradation has an irregular distribution. For example, stains, erosions, graffiti, colour alterations, coherent deposits, darkened, crusts graffiti and so on are degradations that only affect some parts. In these cases, the restoration intervention is located over a surface that isn't the same as that the element modelling. So, knowing the area in which to intervene and its extension is important.

The second way of indicating degradation, in other words, to create an information link about present degradation, it is useful when the degradation is punctual or affects a whole element.

Links are useful to explain problems, in fact, information about degradation extensions, effect description, gravity and urgency decays are included in the report linked.

This approach requires a special study in modelling.

The monument is divided into minimum elements: bricks, stone blocks, wooden boards, and so on. They shall be virtual places where to $\log$ the punctual information. 
The added value of this approach relates to the mapping of the diagnostics performed. In this case, the method seems to be more appropriate. In fact, the indirect investigations such as environmental surveys, thermography, and so on, affect a facade of the monument, so the data can be linked directly to the view, that in the reality has been investigated.

If in a diagnostic campaign, there are direct investigations these are carried out at a precise point and distinctly localizable.

Therefore, the use of minimal, modular, and repeatable elements, to model the monument, allows for the complete preservation of cognitive data comprehensively.

Moving to the specific case analysed in this paper, different approaches have been applied according to the characteristics of data to be linked. In some cases, data comes from specific analysis, and they have a specific data format (numerical values, etc). In other cases, the BIM mapping comes directly from a previously existing mapping (decay or material maps). Of course, these different cases need different approaches even if the workflow is always the same: definition of the smallest element of the model that can "receive" parameters, data assignment (by manual or automatic approach) and data translation to allow the maxim accessibility to the scientific content (not only reserved to high specialized operators).

For example, it was necessary to recover old graphical analyses such as the mapping of degradations developed in last years performed following traditional methods. Otherwise, data were elaborated from previous restoration and consolidation interventions, through links to historical photos, archival reports and so on. In other cases, instead, it was necessary to identify the most appropriate way to manage new information and refer it to the model described above. Choices were made, and algorithms were implemented that facilitated the management of data and their inclusion in the model, thus developing tools that can be used for different purposes.

\subsection{The preservation project of the arch of Augustus in Aosta}

The monument was built contemporary to the foundation of the city: it is an honorary arch since the triumphal arch could be built only in Rome. It commemorates Augusto's victories over the Salassi Celtic tribe. The arch was not inside the city, it was built right after the bridge on Buthier creek, the road crossed under the vault of the arch, leading to the main city gate: Porta Praetoria.

The arch has been modified many times across the centuries, but the first real intervention of restoration dates back only to until the 19 th century.

Between 1883 and 1891, the famous restorer Alfredo D'Andrade oversaw the restoration work. There is very little information about the consistency of his works on Augusto's arch: a set of sketches and drawings of the arch, where he proposed a complete reconstruction of the arch in between stylistic and philological restoration, however, the works has never begun. It is possible that his intervention was limited in small maintenance operations, today not recognizable, and in the removal of an inscription.

Another intervention was done by Ernesto Schiaparelli, probably between 1912-1914. His works on the arch were the most extensive: he changed the roof, dismantled the slates, and changed the gravel fillings, which was causing the collapse of the vault, with a lighter one; the monument was cleaned, and the stone consolidated. The earlier masonry fillings and plaster were removed, missing parts of the decoration were rebuilt with artificial stone, and three capitals were substituted. He filled the gaps with mortar, partially closed the hole in the west niche and, in the vault, secured the integration bricks to the puddingstone with iron bars. Today those bricks and bars are not visible. We do not know if they were removed and substituted with mortar, or they were simply covered in the same period after the pictures were taken.

Those were the last important works done to the monument, during the Second World War the arch was protected with wood scaffoldings.

Until 1961 the road crossed under the arch vault, causing an increasing exposition of the monument to pollution. To solve this problem a roundabout was built around the arch, in 1980 the wooden medieval crucifix was removed and substituted with a copy.

Today a new conservation intervention is necessary and the Superintendency is working to define the guidelines according to the diagnostic analyses of the last years.

\section{RELATED WORKS}

Thematic mapping on HBIM model is a very discussed topic and many aspects have to be considered from the geometric modelling to the linked database.

Some researchers focus on the geometric representation of the themes, by modelling ultra-thin layers overlapping the model elements or concentrating on the informative part, by linking the decay information as parameters on the model objects. This method works well with decays on flat surfaces as in (Matrone et al., 2020)

A similar approach, tested in (Malinverni et al, 2019) gives the possibility to enrich the database with graphic and geometric data that can be effectively used to design and manage future interventions. The advantage is the possibility to associate new ID code that identify the state of conservation of the materials and the detailed description of interventions needed to restore the building. They convert the traditional CAD mapping into parametric BIM objects, by using adaptive components: in this way, the thematic mapping of the material decay can be applied on facades. This enrichment is, above all, related to the graphic representation and to the implementation of the database of BIM model, although it is not clear whether new information can be extracted and how. A very similar approach is used in (Ori, 2021)

(Bruno, Roncella, 2018) deals with the opportunity of an independent database, external to the BIM authoring environment, structured according to a semantic subdivision of the model based on the traditional approach of programmed conservation science.

Another option is to write the themes as flaggable parameters on each model element and the designer flags which themes is present, this method is simple and efficient, but it does not consider the three-dimensional geometry of the mapping. (León-Robles, 2019)

A different approach is, instead, develop by (Croce et al., 2021): by the use of artificial intelligence, they move the state of conservation data (elaborated on a high-resolution orthophoto) from the 2D support (the orthophoto) to a virtual 3D mockup. They developed Surface Information Points (SIPs), similar to hotspot identified over a source model, each one identifying a specific mesh tile, to which information related to the thematic mapping of each tile is linked.

\section{PRELIMINARY RESULTS}

A preliminary, but fundamental step, in this research, has been the definition of the element of maximum granularity, the smallest object of the model that should receive information and 
that, combined in different ways, builds the entire architecture. Relating with historic buildings it is not possible to define a universal granularity, it depends on the objects and the goals. In the case of the Arch of Augustus the smallest element was selected as the ashlar, declined in its various variants (e.g., base ashlar, arch ashlar, etc.) in order to cover all existing cases (Del Pozzo et al., 2022). The real dimension and shape of each ashlar are not fundamental in this part of the work (analytic data processing and parameters management) as the role of each element is to store data and parameters that have to be available for operators and, eventually, can be used for new thematic analysis. Specifically, referring to the Arch of Augustus, the depth of each stone is not really significant, and a generic depth was assumed. Ashlar depth would have been more important if the purpose was the evaluation weight or any structural analysis. Instead, here it is fundamental a correct topology of each element-ashlar respect to the others (Figure 2).

After this first choice, it has been verified the possibility to realize the thematic mappings directly in the BIM Authoring software without the need of a double step (first in CAD, then $\mathrm{BIM}$, as in common practise nowadays). The idea is to develop a thematic mapping view that has a geometric meaning and "lean" it to orthophotos, accurately georeferenced, or directly to the point clouds.

Even if it is not the topic of this paper, it is important to stress that thematic mapping requires a very accurate design phase, in order to correctly highlight the theme to be mapped, to develop eventual databases to be linked with photos of documentation, summary descriptions, standard descriptions, codes and all the elements that are considered necessary to link to the single theme. For the Arch of Augustus, this work has been done for the major part by Aosta Superintendency and its RAVA laboratory (Appolonia et al, 2007) which were in charge of the diagnostic campaign, and they decided the codification and definitions of the themes according to the UNI Normal recommendations, (Idone, 2007).

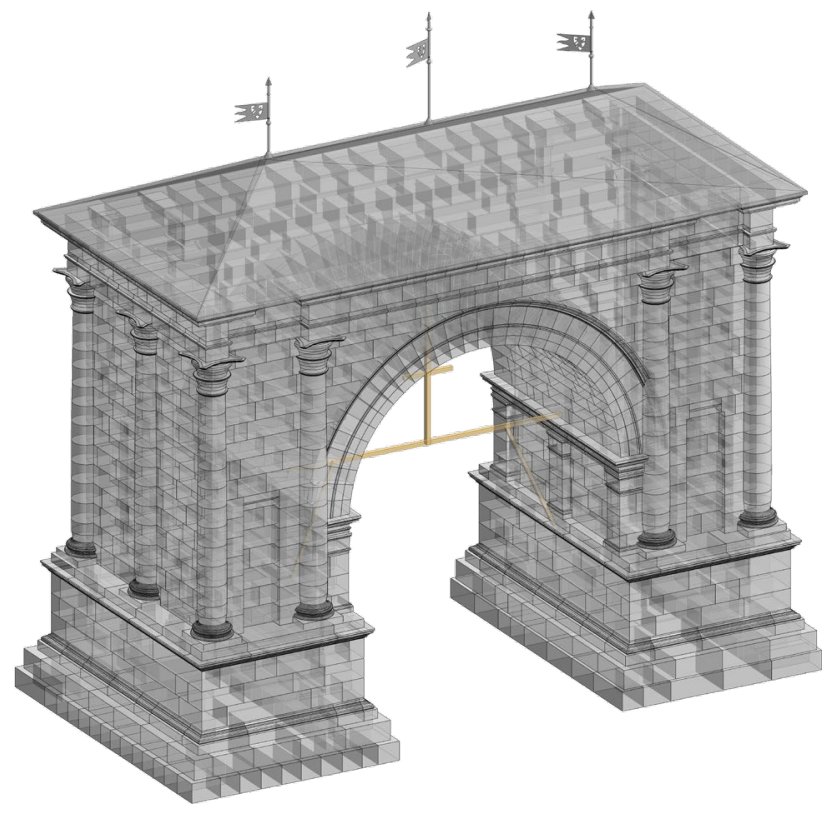

Figure 2 Maximus granularity for the BIM model of the north façade of the Arch.

\subsection{Different thematic views}

In this paper, thematic mapping refers to a graphic representation of a characteristic of the architecture that has to be considered in the preservation design. Typically, they come from the traditional mapping of the restoration process (material, decay, intervention) or from the processing of all data related to the history of the building, its state of damage and specific analyses.

The method suggested in this paper involves the use of various tools native to the Autodesk Revit environment, such as generic models or adaptive models, and project parameters, possibly linked to external databases or internal parameters. The latter, project parameters, can be created and modified ad hoc like generic models.

Using customized project parameters each ashlar (generic model) was then assigned historical information, conservative information, risk assessments and diagnostic. The data coming from these thematism has been translated in numbers, since most of this knowledge is constituted by written scientific essays and reports, or other types of bidimensional reports, written in new project parameters. This translation is essential to allow the user of the BIM system (architect, restorer, designer) to use those data. Moreover, the passage to more comprehensible and immediate numbers allows also to combine the values into new parameters according to specific equations that can be discussed by the operators.

The manual filling could not be avoided, and these types of information do not carry geometric (shape) information and are essentially related to the position of the object. Another approach is discussed in paragraph 3.2 as more related to the proper three-dimensional mapping.

The results have been three thematic views useful for the definition of the preservation project. All data regarding the themes are stored in each ashlar and (thanks to Revit possibility to create rules and filters to changes the visualization style of an object according to specific project parameter values) different thematic mapping can be visualized or extracted automatically.

\subsection{1 "Historical Information" thematic mapping}

The first historical information which is fundamental to design a new preservation project is the knowledge of previous interventions. So a first possible parameter, called Number of Restoration intervention, has the role to summarise, for each ashlar, how many interventions were done. This approach requires manual data insertion and specifically needs to manage the parameter as a flaggable box: the designer or operator flags the box present on each element (León-Robles, 2019). This kind of information is purely binary, it tells only the presence or absenceof previous restoration.

To obtain a more significant view, more parameters have to be used. Each box corresponds to a specific restoration intervention during the history of the Arch, so it is possible to ask different queries: which are the ashars restored in the intervention of D'Andrade between 1883-1891? Or which are the most "restored" ashlars. This allows to keep track in a dynamic way of the elements that underwent more interventions.

The entire system is designed to be very effective and usable by architects, restorers. It is necessary to give the user all the information at disposal in order to verify each single data. For this reason, the historic information parameters are characterized not only by the number of restorations, but also by some links to archival documents such as reports of previous interventions or historic photos (Figure 3).

Of course, according to the restorers, the new designed intervention will become a further element of this thematic map. 


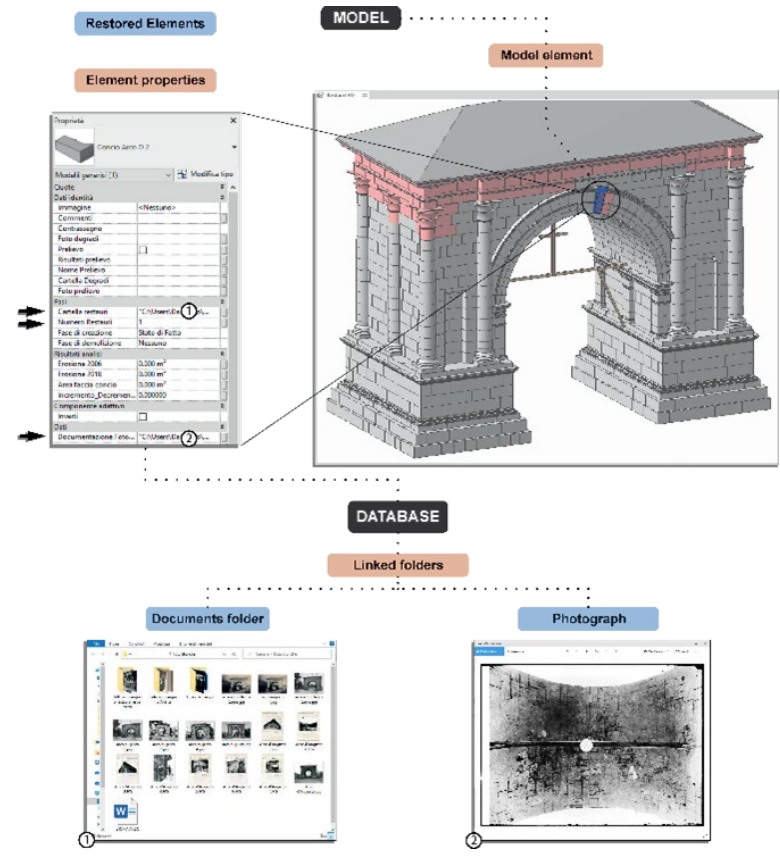

Figure 3: the ashlars coloured in red in this thematic representation have been interested by previous restoration interventions. They are linked to archival data (reports, photos) which allow the user to have specific details.

\subsubsection{Diagnostic}

Another thematic view is concerning all the diagnostics done by the RAVA laboratory of Superintendency. Many of those analyses started from a single sample which allowed to extract punctual information and to extend the evaluation to the entire area. In this case the three-dimensional information is the position of the samples. The system is the same used until now, since sampling is an invasive operation which has been done only once (and generally is not repeated), so a single flaggable box parameter is sufficient to record the event.

If an ashlar has been sampled there will be an additional parameter with the Sample name. Access to the original documentation and to the raw data is guaranteed by $U R L$ parameters. These parameters can open the folders containing the analysis results, the scientific reports and the description of the diagnostic procedures. (Figure 4).

\subsection{Dynamic mapping from previous maps}

A completely different thematic mapping is concerning the presence of decay on the facades. The main difference is that the starting point is already the result of elaboration and above all this theme is presented not through numbers, but through a graphic scheme. The original maps were done in AutoCAD .dwg by drawing the contours of decay effects over the orthophotos: data refer to two mapping campaigns, done in 2007 and in 2018.

The goal of this part was a little bit different from the others sections. The first aim was to import thematic mapping without the necessity of manual re-drawing of the areas. But the second, more advanced, study regards the comparison between the two campaigns.

To solve this problem, the workflow has been divided into different steps. In the first step, the 2D shape of pathology must be projected on the 3D model, and divided by the monument ashlar, to find the single area insisting on the ashlar, in the second the first step is iterated for the other mapping and the two areas compared.

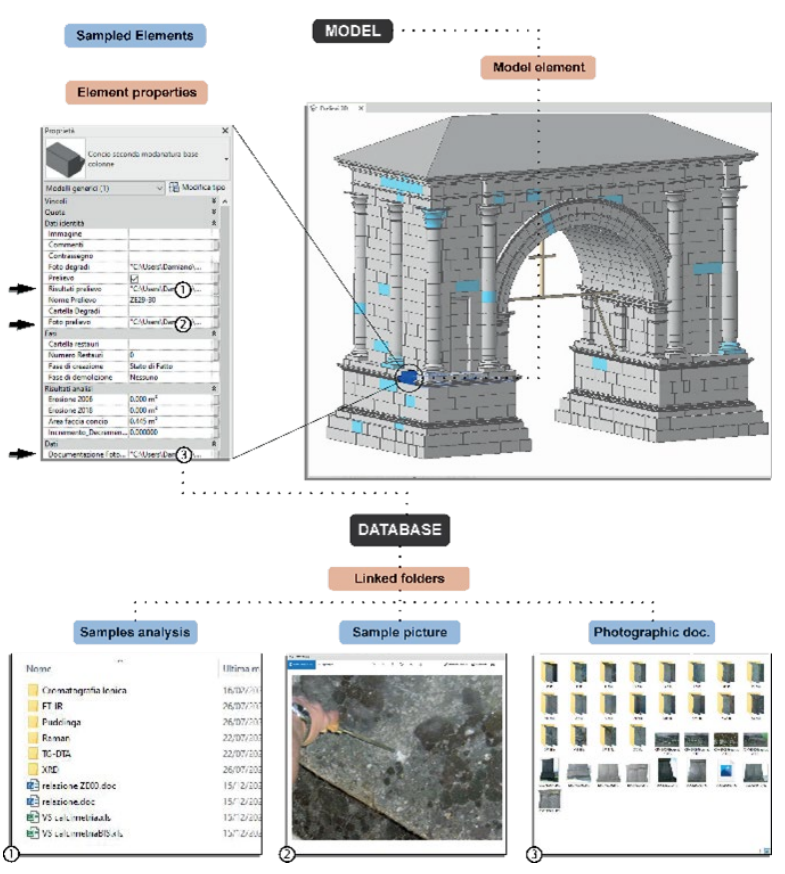

Figure 4: the blue ashlars are the location of samples for the different analysis made by RAVA laboratory. On the bottom part, all the types of documents connected with the single element by the URL to allow a direct link

Since there isn't a command available to do so it was necessary to work with Dynamo, Revit Visual Programming application to create a script able to perform our requests.

The starting material was 2D drawings done with Autodesk AutoCAD. They were structured as traditional digital mapping: many layers, each one for a single decay, and in every layer, there are polylines to describe the position over the façade. Of course, according to the fact that they belong to the same work environments, these files could be imported in Revit and read without compatibility issues.

Although it seems obvious, it must be emphasised that the basic condition for proceeding with this workflow is that all drawings are georeferenced in the HBIM model reference system. This can be achieved directly if the survey is designed, and conducted, based on the same topographic network. Or it requires a rototranslation operation of the $\mathrm{CAD}$ drawing to georeference it into the chosen reference system.

To compute the two decays areas the software needs two number parameters, which were labelled with the pathology name and the year of the survey; other two numerical parameters were created for later operations, one for the Area (ashlar surface) and one for Increase/Decrease (expressed as percentage)

Despite the fact that finding the intersection between two elements (the 2D decay projected on the 3D surface) seems easy, the process turned out not to be that simple and many workaround solution have been used.

Direct 2D projection onto 3D surface did not work, the next try was to create 3D extrusion (generic model) of the 2D mapping and intersect it with the generic models of the ashlars. 
With Revit 2021 it was possible to select directly in Dynamo the drawing files, in .dwg format, and create in Dynamo (not in the model space) the corresponding 3D extrusion as generic models and intersect it with the Arch model. With the update to Revit 2022, this function was no longer supported and until further update the .dwg files have to be imported in Revit and the extrusion created manually.

The final Dynamo script selects the 3D mapping solid, the element of the corresponding façade and run a clash detection. The intersected ashlars are filtered, in this stage, they are only elements, so their 3D geometry properties need to be retrieved, additional commands creates a solid of the intersection only.

This single solid spanning across all the façade is then cut to obtain the unitary intersection on each ashlar. Now the next operation is the explosion of the intersection solid faces. With a series of functions, involving the computation of surface normal, it was possible to select only the outward surfaces (the software recognizes all the surfaces, also the inner one) and calculate, finally, the pathology area on the ashlar which has been written in the dedicated parameter.

The script calculates also the ashlar surface area and writes it in the parameter of the ashlar. Of course, these operations are done for each kind of decay, so at the end, every element has a certain number of Boolean parameters corresponding to the decays, and for some of them also the area (Figure 5).

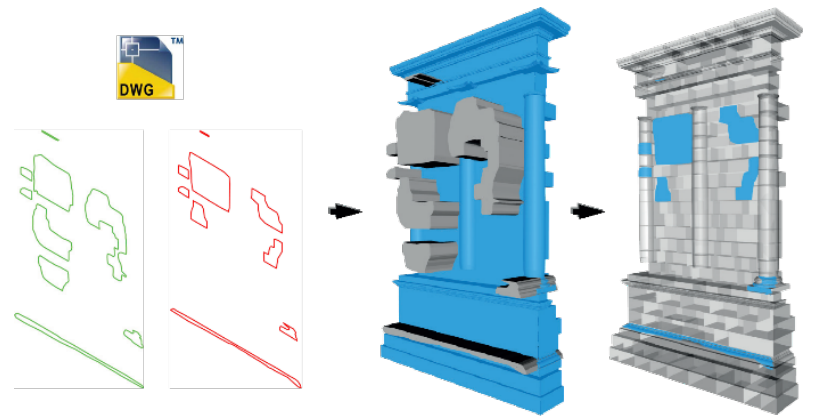

Figure 5: from the left the contours of the decay in CAD environment (the two different colors refer to different decays). Then, in the centre, the extrusion of the theme and the clash detection between the solid of the decay and the solid of the ashlar.

The second part of the Dynamo script is developed in order to make the comparison between the two different campaigns of decay mapping. In this case, the script does not work on the geometry, but it reads the area-parameter of the same decay make a subtraction and express is at a percentage respect the global surface area. To these percentages interval is possible to assign a different colour, this process transforms 2D drawings into a 3D mapping describing a behaviour over time (Figure 4).

\subsubsection{Conservation and risk assessment}

At the end of the mapping transfer process, it is possible to visualise specific themes on the model.

However, the restorer, who is in charge of preparing the conservation project, has also the task of synthesising all the analyses, diagnoses and observations into a single system. This operation of re-elaboration and synthesis becomes a further step in which the result, graphically similar to the other thematic representations, is the result of the definition of a scale of gravity of each effect and each degradation (Figure 6).
This operation requires the intellectual and critical intervention of the restorer, but it can be carried out for each individual ashlar, working with numerical values that are appropriately combined and weighted.

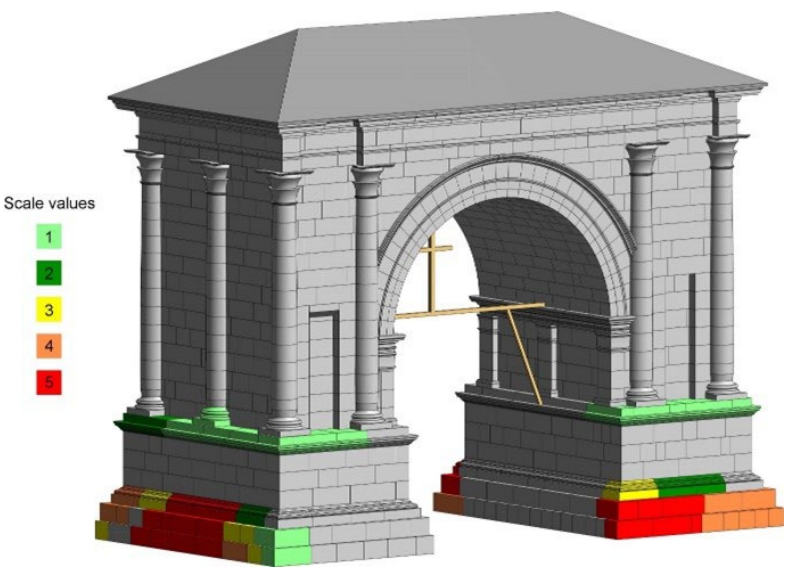

Figure 6: Thematic view about conservation and risk assessment. This view is the outcome of the restorer evaluation all about the arch, its state of conservation and, more generally, its exposure to risk.

\section{DISCUSSION}

These first tests made on the Arch of Augustus highlighted the potential of this approach, both in operational terms and in terms of quality. In fact, it allows the use of existing data (in various formats), not only with a view to a data reuse, but also in function of a temporal comparison, useful to quantify, for example, the progress of an alteration or a state of degradation of a specific ashlar.

The preliminary results are promising, however, some problems arise, related to the fact that working in $3 \mathrm{D}$ space is complex and creating too many thematic views and related parameters could be confusing, especially when attempting to fully exploit the three-dimensional content of survey data in the BIM environment.

As regards the risk assessment mapping (par. 3.2.1) it can be argued that requiring a critical analysis of ashlar by ashlar and having a high number of $3 \mathrm{D}$ views for each degradation, requires too much effort and is difficult to manage.

In this paper, we proposed a tool that tells which pathologies are endangering the monument most, and on which part of the monument are found.

This allows to plan works for functional lots based on pathologies groups, with similar treatment or similar urgency level; If simplification is wanted, the critical analysis can be done with a pure risk factor, (0-5) mediator of all the pathologies present.

To not lose the information of which pathologies are present on that ashlar, the parameters will change to flaggable box parameters, (León-Robles, 2019) resulting in one risk indicator, and a list of pathologies present. Which path adopt must be decided with the clients and the designers, considering their needs and their resources.

Some issues were raised according to the first part of the script of mapping projection in BIM environment (par. 3.2) The first one is the projection of a $2 \mathrm{D}$ geometry on rounded elements, such as the columns. Starting from two facades, in a relative 
quasi-orthogonal position, the two projections on a single circular column produce a double reading, two different values from two $2 \mathrm{D}$ views are projected on the same $3 \mathrm{D}$ element (Figure 7, left).

Another issue that will affect the exact area calculation is the failure to recognize all the surfaces of an ashlar. The normal vector method employed earlier cannot distinguish the horizontal surfaces that are part of the objects from the other faces which are in contact (figure 7 right). Always for this reason the horizontal area between two offset ashlars is not calculated (Figure 6) As for the earlier issue, this will affect only the exact area calculation. Is not a huge issue because, in a sense, this is similar to what happens also in professional practice where the mappings, carried out on the average plane of the facade, are then multiplied by a safety factor that takes into account all the rounding and the difficulty of managing eventual indentations (Figure 7, right)

The final goal is to automatically compare two decay surveys and find the increase or decrease of a pathology; this calculation will not be severely affected since the $2 \mathrm{D}$ geometry projected are just two views of the same 3D real element, and the little parts of the horizontal surface are not present also in the $2 \mathrm{D}$ drawings.
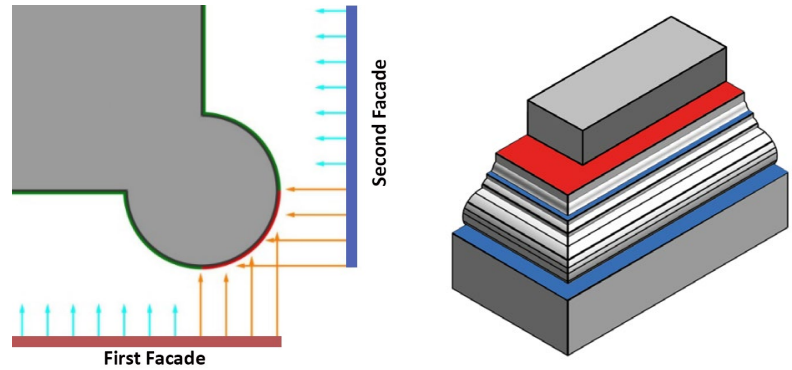

Figure 7 (On the left) the issue of double projection on the curve surface of the column along the corner. (On the right) the horizontal elements of the object with part of their area which which is calculated incorrectly because it is in contact with other surfaces.

There have been also a few stabilities issues, the ashlars whose shape is based around a revolution (column, capitals) are recognized, but not all of them, and not always the same, the unrecognized elements change every time the algorithm is run. However, these problems are a minor setback compared to what it was possible to achieve with this script, unrecognized parts can be fixed after the testing phase and with a more advanced version of the algorithm.

\section{CONCLUSIONS}

Two approaches can be followed to create HBIM mappings, one numerical, for those themes hard to express with graphic tools, and one theme-object oriented, that could be applied also to a brand new thematic mapping, provided that can be graphically identified. The thematism can be drawn directly in Revit, its graphic boundaries can be recognized automatically with the script realized in the Autodesk Dynamo environment, transform them into generic models with specific parameters (for example the theme of the mapping) verify if they intersect with the single objects (the ashlars). If so, it is possible to quantify the intersection surface which, barring some errors, can be considered the area of the object affected by that particular theme.
Through these algorithms, developed in Dynamo, it is, therefore, possible on one hand to optimize the data entry process and on the other hand, if the minimum element of the model is carefully chosen, also to link the thematism to the whole model.

Although the method presented was correctly applied to the selected case study, it is important to discuss and further explore the fact that the process is not completely optimized. Furthermore, from this research arise the urgent need for the development of interdisciplinary working groups (surveyor architects, conservator architects, computer scientists and database experts), in order to effectively overcome the dichotomy 2D 3D. This will allow exploiting the 3D content of the point clouds, without having to necessarily flatten it on a reference plane.

\section{REFERENCES}

Adami A., Appolonia L., Scala B., 2021. The Arch of Augustus in Aosta: data and analysis reuse. In Proceedings of the joint international event 9th ARQUEOLÓGICA 2.0 \& 3rd GEORES, Valencia (Spain). 26-28 April 2021 CGAL, 2021. Computational Geometry Algorithms Library, http://www.cgal.org (Last accessed: Oct 2021).

Adami A., Appolonia L., Scala B., 2021. The Arch of Augustus in Aosta: data and analysis reuse. In Proceedings of the joint international event 9th ARQUEOLÓGICA 2.0 \& 3rd GEORES, Valencia (Spain). 26-28 April 2021 CGAL, 2021. Computational Geometry Algorithms Library, http://www.cgal.org (Last accessed: Oct 2021).

Appolonia, L., Migliorini, S., Idone, A., Piccirillo, A. ,2007. L'Arco di Augusto in Aosta: un esempio di percorso programmato tra progettazione e diagnostica. In Bollettino della Soprintendenza per $i$ beni e le attività culturali, 4, pp. 215-228

Bruno, N. and Roncella, R., 2018. A Restoration Oriented Hbim System for Cultural Heritage documentation: the case study of Parma Cathedral, Int. Arch. Photogramm. Remote Sens. Spatial Inf. Sci., XLII-2, 171-178, https://doi.org/10.5194/isprsarchives-XLII-2-171-2018, 2018

Croce, V., Gabriella Caroti, g., Piemonte, A., 2021 Propagation of semantic information between orthophoto and 3D replica: a H-BIM system for the north transept of Pisa Cathedral, Geomatics, Natural Hazards and Risk, 12:1, 2225-2252, DOI: 10.1080/19475705.2021.1960432

Del Pozzo, D., Appolonia, L., Scala, B., Adami A., 2022. Federated HBIM models for cultural heritage: survey model and conceptual model. In proceedings $3 d A r c h 2022$ (in print)

Frutaz F.G., 1920, L'arc d'Auguste et sa restauration, in Augusta Praetoria: revue valdôtaine de pensée régionalistes, No. 1-2, pp.17-26.

Idone A., 2007, L'arco di augusto in Aosta: un esempio di percorso programmato tra progettazione e diagnostica, tesi di Laurea Triennale in Scienza e Tecnologia per i Beni Culturali, università degli studi di Torino Facoltà di scienze Matematiche Fisiche e Maturali.

León-Robles C., Reinoso-Gordo J., González-Quiñones J., 2019, Heritage building information modeling (H-BIM) applied 
to a stone bridge, in ISPRS International Journal of GeoInformation, No. 8(3), p. 121.

Malinverni, E. S., Mariano, F., Di Stefano, F., Petetta, L., and Onori, F., 2019. Modelling In HBIM to document materials decay by a thematic mapping to manage the Cultural Heritage: the case of "Chiesa Della Pietà" in fermo, Int. Arch.

Photogramm. Remote Sens. Spatial Inf. Sci., XLII-2/W11, 777784, https://doi.org/10.5194/isprs-archives-XLII-2-W11-7772019,2019

Matrone F., Santo R., 2020, La fruizione dei dati da modelli HBIM, in Architectural Design and Studio - HBIM e Geomatica per $i$ beni culturali, Franco Angeli, Milano, pp. 94

Ori L., 2021, Ottimizzazione della metodologia HBIM applicata al progetto di restauro architettonico, in HBIM e Geomatica per $i$ beni culturali, (edited by A. Adami), Franco Angeli, Milano, 2021, pp 110-127. 